\title{
Adhesions of pacing catheter to tricuspid valve: adhesive endocarditis
}

\author{
H. DAVID FRIEDBERG AND GEORGE F. D'CUNHA \\ From the Veterans Administration Center, Wood, Wisconsin, and the Marquette School of Medicine, \\ Milwaukee, Wisconsin, U.S.A.
}

\begin{abstract}
A case is reported in which the presence of endocardial pacemaker catheter electrodes provoked an unusual reaction-an adhesive endocarditis. Two pacemaker catheters were in the right ventricle during the last nine months of life. The normal reaction to these foreign bodies of fibrin deposition and endothelialization was intensified so that the first catheter could not be removed after 17 days. Because one catheter was introduced from the left side of the neck, it lay in a commissure of the tricuspid valve to which it was firmly adherent. This constitutes a potential hazard if the removal of the catheter is forcibly attempted, although it does not interfere with the function of the tricuspid valve. Despite this, thrombosis was absent and generally is not a problem with modern materials.
\end{abstract}

Transvenous pacing is now an established treatment for chronic heart block. The effects of the long-continued presence of a foreign body in the bloodstream and in the heart cavity are largely unknown and have occasioned some thoughtful concern (Youmans, Derrick, and Wallace, 1967 ; British Medical Journal, 1968). We report, therefore, an unusual complication of transvenous pacing which has not been described before, and which reflects the reaction of the endocardium to the catheter.

\section{CASE SUMMARY}

A 54-year-old white man had suffered recurrent attacks of Stokes-Adams syncope due to high-grade atrioventricular block. On 17 July 1967 a unipolar catheter electrode was implanted into the right ventricular endocardium via the right external jugular vein. It became necessary to replace the entire system with one of another design. On 3 August 1967, a bipolar electrode was implanted via the left external jugular vein. It was not possible to remove the first catheter. Pacing was satisfactory until the patient died of a massive cerebral haemorrhage on 13 April 1968, i.e., nine months after the first pacing catheter had been inserted. At necropsy the tip of the unipolar catheter was firmly embedded in the right ventricular apex, and the intraventricular portion of its body was adherent to the wall and covered with endothelium (Figure, arrowed). Higher up, this catheter was free of the tricuspid valve. The second bipolar catheter lay differently in the right ventricular cavity. Its tip was also embedded in the apex but most of its body was free. It was firmly adherent to the tricuspio valve, nestling in the commissure (Figure). Thrombu品 was absent.

\section{COMMENT}

Soon after a catheter has been placed within the heart, a thin layer of fibrin is deposited which. does not get thicker with the passage of time (Lagergren, Dahlgren, and Nordenstam, 1966 Parsonnet, Zucker, Kannerstein, Gilbert, and Alvares, 1966). Presumably fibrinolysis and fibring deposition reach an equilibrium. Later an endo thelial lining envelops the catheter and the smalp risk of thrombosis is reduced or eliminated. While raw fibrin is present upon the catheter it may adhere to nearby structures. With the advent of endothelialization, the catheter is incorporatedE. into the structure of the heart. Such incorporations is the means of fixation of the catheter to the apex. It is tempting to surmise that in somp patients fibrin deposition or removal is abnormalcu accounting for late displacements of the cathetes tip. In this man, the adhesive reaction seems tof have been more intense than normal.

The unusual path of the second bipolar ${ }^{+}$ catheter allowed its peculiar adhesion to the tricuspid valve. It made a large rightward swee ${ }_{D}$ from the left side of the neck through the extrem? right side of the tricuspid valve before turning back to the left to reach the apex. Thus, most this catheter lies free in the cavity. Adhesion of the catheter to the tricuspid valve is not necese 
FIGURE. Two catheters in situ in the right ventricle at necropsy. The unipolar catheter, arrowed, is almost totally incorporated into the septal wall and apex. The bipolar catheter $(B C)$ is enclosed within a fold of tissue covered with endothelium and attached to the tricuspid valve (TV).

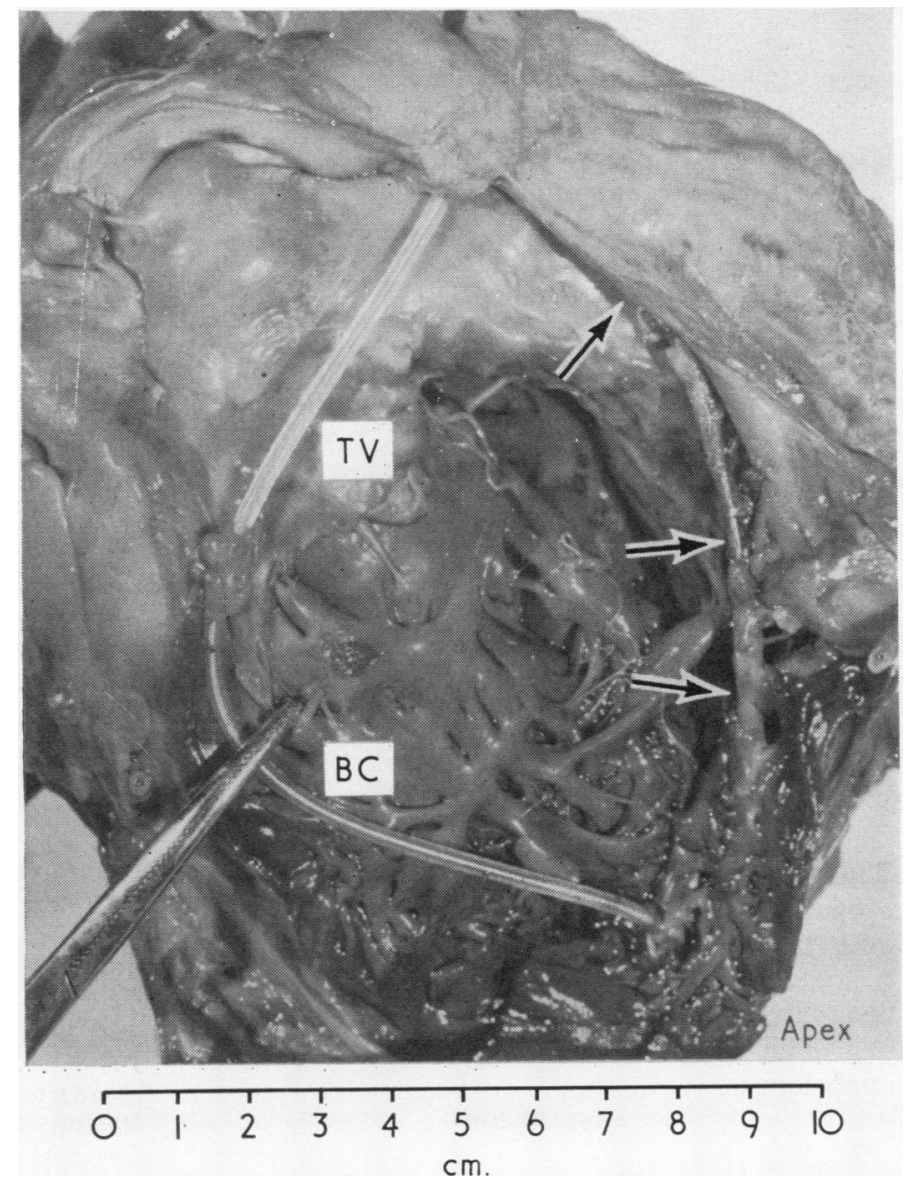

sarily harmful. Because this structure is large, floppy, and almost redundant, the leaflets wrap themselves around the intruder and prevent regurgitation of blood. Thus, with both catheters a marked adhesive endocarditis is seen. This is an early phenomenon, for the first catheter could not be removed after only 17 days in situ. Vigorous attempts at removal of a catheter can be seen to harbour great dangers. It is noteworthy that, despite the intensity of the adhesive reaction in the endocardium, thrombosis was absent. Rapid blood flow and early endothelialization mitigate against thrombosis.

\section{REFERENCES}

British Medical Journal (1968). Long-term cardiac pacing (Leading article). Brit. med. J., 2, 2.

Lagergren, H., Dahlgren, S., and Nordenstam, H. (1966). Cardiovascular tissue response to intracardiac pacemaking. Acta chir. scand., 132, 696 .

Parsonnet, V., Zucker, I. R., Kannerstein, M. L., Gilbert, L., and Alvares, J. F. (1966). The fate of permanent intracardiac electrodes. J. surg. Res., 6, 285 .

Youmans, C. R., Derrick, J. R. and Wallace, J. M. (1967). Considerations of complications of permanent transvenous pacemakers. Amer. J. Surg., 114, 704. 